\title{
Impact of new frequency standards on the international time scales
}

\author{
E. Felicitas Arias ${ }^{1,2}$ and Gianna Panfilo ${ }^{1}$ \\ ${ }^{1}$ International Bureau of Weights and Measures, \\ Sèvres, France \\ email: farias@bipm.org \\ ${ }^{2}$ Associated astronomer at the Paris Observatory, \\ Paris, France
}

\begin{abstract}
The reference time scales maintained at the International Bureau of Weights and Measures (BIPM) are constructed with data from industrial clocks and primary frequency standards operated in national metrology laboratories and observatories world-wide distributed. Clocks are compared making use of techniques of time transfer between remote sites. The algorithm of calculation relies on clock weighting and clock frequency prediction. We briefly present hereafter the influence of some clocks on the scales, as well as the possibilities for improvement.
\end{abstract}

Keywords. atomic time scales, frequency accuracy and stability, atomic standards

\section{Summary}

The statistical treatment of industrial clock data (frequency prediction, weighting procedure) is designed to assure the high frequency stability of the scale on an averaging interval of 30 days (BIH 1974), (Guinot \& Thomas 1988), (Audoin \& Guinot 2001). The introduction of measurements of primary frequency standards, together with a strategy for steering the frequency of the scale contribute to improving its frequency accuracy.

International atomic time (TAI) and Coordinated Universal Time (UTC) are calculated from monthly blocks of clock data and measurements of primary frequency standards over one year. The resulting atomic time scale has long-term instabilities that make in unsuitable for applications such as timing of millisecond pulsars. Another time scale, TT(BIPMYY) (Petit2003), (Petit 2008), is calculated every year with a different algorithm that makes use of all existing data from primary frequency standards, and the resulting scale is not affected by the instabilities of TAI.

In the last five years more caesium fountains, which realize at best the second of the international system of units (SI) started contributing data to the BIPM. The statistical uncertainty of these realizations of the second is of order $10^{-16}$ (BIPM 2009). The frequency steering tries to compensate, in a monthly process and without degrading the stability of the scale, a drift of the frequency observed between the fountains and the beam standards. When compared to TT(BIPM), the frequency of TAI shows a drift of 4 parts in $10^{16} /$ month. The origin of this drift is not completely known, but studies under development seem to indicate that an incomplete model of frequency prediction of clocks in the algorithm of calculation of TAI could be partially responsible for this effect. A linear frequency prediction algorithm is applied to both, caesium industrial beams and hydrogen masers; these later represent $12 \%$ of the clock weight in TAI. While the linear model is well adapted to caesium standards, it fails to represent the frequency drift of hydrogen masers. Tests using a quadratic frequency prediction for hydrogen masers show that this model represents better the hydrogen maser's drift, and indicate that about 
$20 \%$ of the drift could be provoked by the inappropriate frequency model (Panfilo \& Arias 2009).

While the caesium provides the primary realization of the SI second, other radiations in the microwave and optical frequencies have been recommended as secondary representations of the second, and they are called to provide in the near future primary realizations (Gill \& Riehle 2006). These frequency standards have already proved to be one order of magnitude more accurate than the caesium fountains, and for their utilisation it will be necessary to make use of highly accurate frequency transfer techniques.

\section{References}

Audoin, C \& Guinot, B. 2001, The measurement of time, Cambridge University Press Bureau International de l'Heure 1974, BIH Annual Report for 1973, Observatoire de Paris Bureau International des Poids et Mesures 2009, BIPM Annual Report on Time Activities for 2008, 26

Gill, P. \& Rihele, F. 2006, Proc. $20^{\text {th }}$ EFTF, 282

Guinot, B. \& Thomas, C. 1988, Annual Report of the BIPM Time Section, 1, D1-D22

Panfilo, G. \& Arias, E. F. 2009, Special issue of UFFC, submitted

Petit, G. 2003, Proc. $35^{\text {th }}$ PTTI, 307

Petit, G. 2008, Proc. $7^{\text {th }}$ Symposium on Freqency standards and metrology, in press 